\title{
Prevalência de sequelas neurológicas associadas a trauma em face
}

\author{
Prevalence of neurological sequelae associated with facial trauma
}

\author{
Cassian Taparello* \\ Kássia Estefânia Hauck ${ }^{* *}$ \\ Tiago Nascimento Mileto ${ }^{* * *}$ \\ Ricardo Scariot ${ }^{* * *}$ \\ Silvana Gonçalves de Almeida ${ }^{* * * *}$ \\ Ferdinando de Conto ${ }^{* * * * *}$
}

\section{Resumo}

Introdução: pacientes acometidos por traumatismo craniofacial podem evoluir com sequelas variadas. Objetivo: identificar a prevalência de sequelas neurológicas ocorridas entre os pacientes com traumatismo craniofacial em um serviço de referência para o trauma no sul do Brasil. Sujeitos e método: foram analisados 1.385 prontuários, em que 169 (12\%) pacientes foram selecionados com trauma em crânio e face simultaneamente, levando em consideração o agente etiológico, a procedência, a idade, o sexo do paciente e a localização das fraturas. Resultados: uma taxa de $85 \%$ dos indivíduos era do sexo masculino, com faixa etária entre 31-40 anos. Os fatores etiológicos mais prevalentes foram acidentes de trânsito (36\%), quedas (22\%) e violência interpessoal (21\%). No grupo selecionado, o traumatismo cranioencefálico esteve presente em $89 \%$ dos casos; em $64 \%$ da população, não houve sequela neurológica; $28 \%$ apresentaram algum tipo de sequela; e em $8 \%$ dos prontuários não havia informações completas. Conclusão: na população estudada, mesmo com um subgrupo específico de traumatizados, houve prevalência de gênero masculino, terceira década de vida e acidente automobilístico. Além disso, a região anatômica mais acometida foi o conjunto de ossos do terço médio da face, e, ainda nesta condição de associação, o traumatismo cranioencefálico esteve presente na maioria dos casos, porém, somente $28 \%$ dos casos evoluíram com alguma sequela neurológica.

Palavras-chave: Epidemiologia. Traumatismos maxilofaciais. Traumatologia.

\section{Introdução}

O trauma em face não apresenta riscos apenas estéticos e locais ao paciente, pois, dependendo da energia da agressão, o cérebro também pode ser afetado, ocasionando, assim, sequelas sistêmicas ${ }^{1}$.

Lesões em região da cabeça podem danificar e comprometer a fala, a deglutição, a voz, a audição, dentre outras estruturas importantes do corpo humano $^{2}$. Isso afeta a vida social das pessoas que são acometidas, podendo até interferir na capacidade de se relacionar com os outros.

O número de casos de traumatismo craniano vem aumentando nos últimos anos nos países em desenvolvimento, sendo uma das principais causas de morbidade e mortalidade em adultos jovens. Sem dúvida, trata-se de um problema de saúde pública ${ }^{3}$.

Uma das principais causas de trauma de face e traumatismo craniano é ocasionada por acidente automobilístico, envolvendo automóveis, motocicletas, bicicletas e pedestres. Isso decorre, geralmente, de traumas de alta cinemática. Além disso, aproximadamente um terço dos traumas em face apresenta lesões intracranianas ${ }^{4}$.

Nos últimos anos, têm-se aprofundado os estudos no intuito de se buscarem os fatores prognósticos para o traumatismo cranioencefálico (TCE). A gravidade inicial medida pela escala de coma Glasgow tem sido o instrumento mais usado para avaliação do prognóstico funcional após o TCE, e uma menor pontuação nessa escala está relacionada à

\footnotetext{
Cirurgião-dentista. Residente em Cirurgia e Traumatologia Bucomaxilofacial (HCPF/UPF/SMSPF), Faculdade de Odontologia, Universidade de Passo Fundo, Passo Fundo, Rio Grande do Sul, Brasil.

Cirurgiã-dentista. Especialista em Cirurgia e Traumatologia Bucomaxilofacial. Mestranda em Clínica Odontológica, Faculdade de Odontologia, Universidade de Passo Fundo, Passo Fundo, Rio Grande do Sul, Brasil.

* Cirurgião-dentista. Residente em Cirurgia e Traumatologia Bucomaxilofacial (HCPF/UPF/SMSPF), Faculdade de Odontologia, Universidade de Passo Fundo, Passo Fundo, Rio Grande do Sul, Brasil.

Passo Fundo, Rio Grande do Sul, Brasil.
Acadêmico de Odontologia, Faculdade de Odontologia, Universidade de Passo Fundo, Passo Fundo, Rio Grande do Sul, Brasil.

${ }^{* * * * * *}$ Bacharel em Estatística, Centro de Ciências Naturais e Exatas, Universidade Federal de Santa Maria, Santa Maria, Rio Grande do Sul, Brasil.

${ }^{* * * * * *}$ Coordenador do Serviço de Residência em Cirurgia e Traumatologia Bucomaxilofacial (HCPF/UPF/SMS-PF), Faculdade de Odontologia, Universidade de Passo Fundo, Passo Fundo, Rio Grande do Sul, Brasil.
} 
maior gravidade dos traumatismos cranioencefálicos e, consequentemente, é um dos principais fatores relacionados à pior prognóstico ${ }^{5}$.

Este estudo tem como objetivo identificar a prevalência de sequelas neurológicas ocorridas entre os pacientes diagnosticados com trauma craniofacial pelo Serviço de Cirurgia e Traumatologia Bucomaxilofacial no Hospital São Vicente de Paulo (HSVP), em Passo Fundo, Rio Grande do Sul, Brasil.

\section{Sujeitos e método}

Foram analisados 1.385 prontuários de pacientes atendidos pelo Serviço de Cirurgia e Traumatologia Bucomaxilofacial do Hospital São Vicente de Paulo, em Passo Fundo, Rio Grande do Sul, dos quais 169 (12\%) foram selecionados por apresentar histórico de trauma em crânio e face simultaneamente, ocorridos entre os anos de 2000 a 2012. Os dados avaliados consideraram: agente etiológico, procedência, idade, gênero do paciente e localização do trauma.

Os agentes etiológicos foram divididos em sete grupos: acidentes de trânsito, quedas, violência interpessoal, atropelamentos, acidentes desportivos, outros e não informados. Acidentes com animais e acidentes domésticos foram atribuídos ao grupo "outros". Prontuários que não continham informações foram atribuídos ao grupo "não informados".

A localização das fraturas foi dividida em sete grupos: ossos malares, osso mandibular, órbita, nariz, osso maxilar, fratura tipo Le Fort (incluindo I, II e III, devido à baixa incidência neste estudo) e fraturas complexas (quando o indivíduo apresenta mais de um osso acometido pelo trauma). Fraturas dentoalveolares foram incluídas em fraturas mandibulares ou maxilares, dependendo da localização.

A classificação das injúrias associadas ao trauma facial foi dividida em: membros superiores, membros inferiores, crânio (representado por TCE), tórax, coluna vertebral, abdômen e escoriações. Casos em que não havia injúrias foram incluídos no grupo denominado ausente.

O projeto foi submetido e aprovado no Comitê de Ética em Pesquisa da Universidade de Passo Fundo, sob protocolo número 0159039800011.

\section{Resulltados}

Dos 1.385 prontuários avaliados, $12 \%$ apresentavam alguma injúria relacionada a trauma craniofacial, equivalendo a 169 prontuários (Figura 1). Dos 169 pacientes avaliados, $85 \%$ eram do sexo masculino e apenas $15 \%$ do feminino. Em relação à procedência dos pacientes, $38 \%$ residiam em Passo Fundo, RS.
Figura 1 - Análise de prontuários

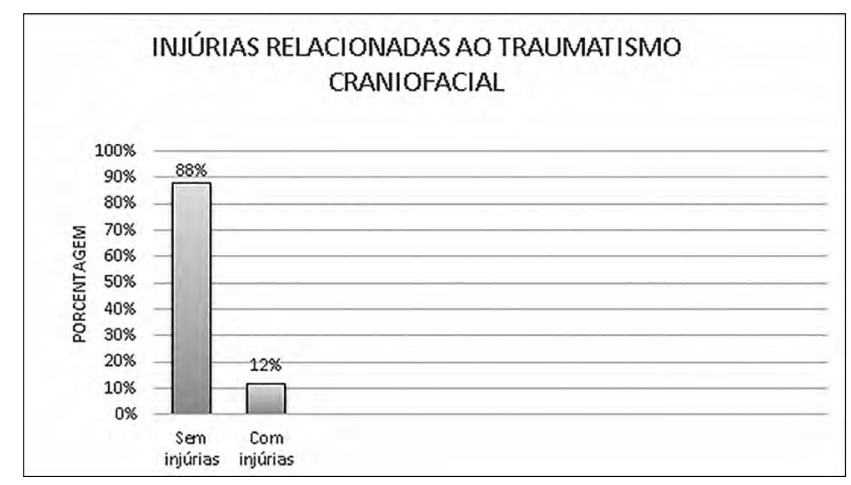

Fonte: autores.

Este estudo observou a distribuição dos pacientes avaliados por faixa etária. Assim, 28\% dos pacientes encontravam-se na faixa de $20-29$ anos; $17 \%$ entre 30-39 anos; e 17\% entre 40-49 anos (Figura 2).

Figura 2 - Faixa etária dos pacientes avaliados

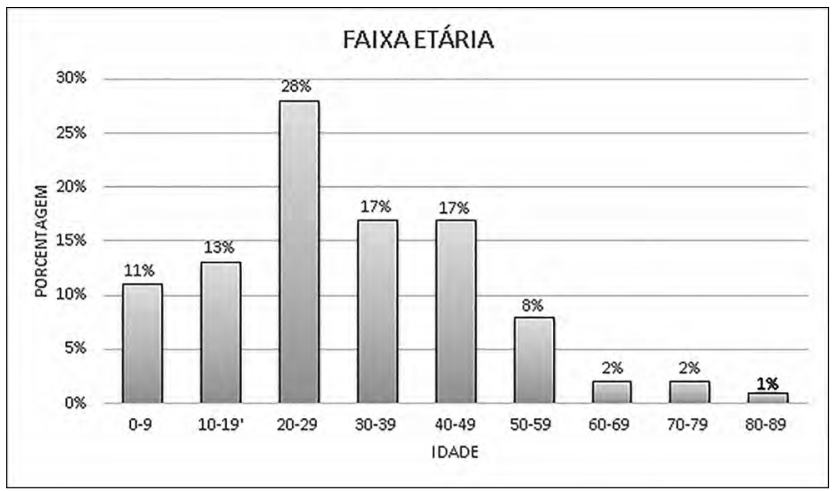

Fonte: autores.

Os agentes etiológicos observados estão representados na Figura 3 e compreendem: acidentes automobilísticos (36\%); quedas (22\%); violência interpessoal (21\%); não informado (9\%); outros (6\%); atropelamentos $(4 \%)$; e esportes $(2 \%)$.

Figura 3 - Agentes etiológicos dos pacientes avaliados

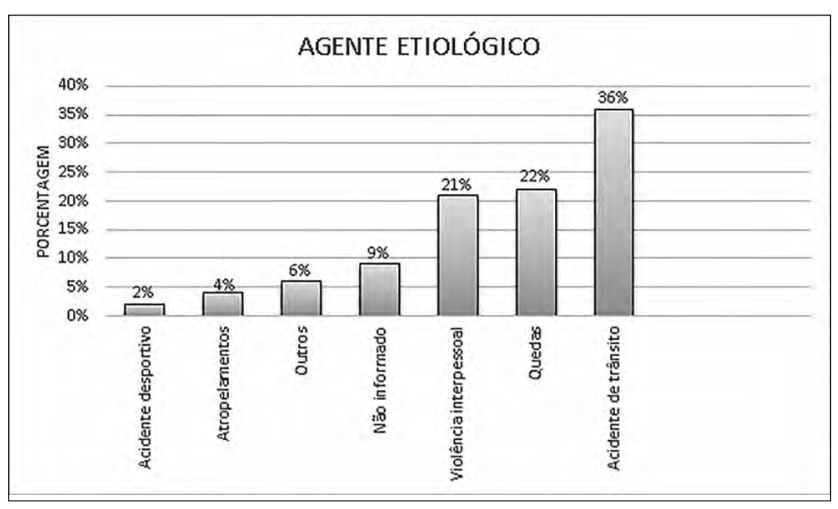

Fonte: autores. 
Na Figura 4, observa-se a distribuição dos pacientes em relação ao sítio acometido, que compreende: fraturas complexas (29\%); mandíbula (22\%); órbita (20\%); ossos malares (15\%); nariz (15\%); fraturas Le Fort (11\%); e maxila (4\%).

Figura 4 - Local acometido dos pacientes avaliados

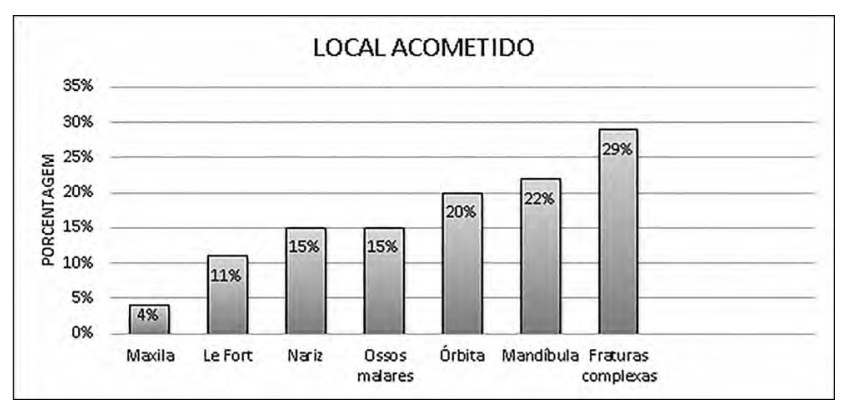

Fonte: autores.

As injúrias observadas são apresentadas na Figura 5 e compreendem: TCE (89\%); escoriações (4\%); lesões em membros inferiores (3\%); lesões em membros superiores $(2 \%)$; tórax $(1 \%)$; coluna vertebral (1\%); e abdômen (1\%).

Figura 5 - Injúrias associadas ao trauma em face

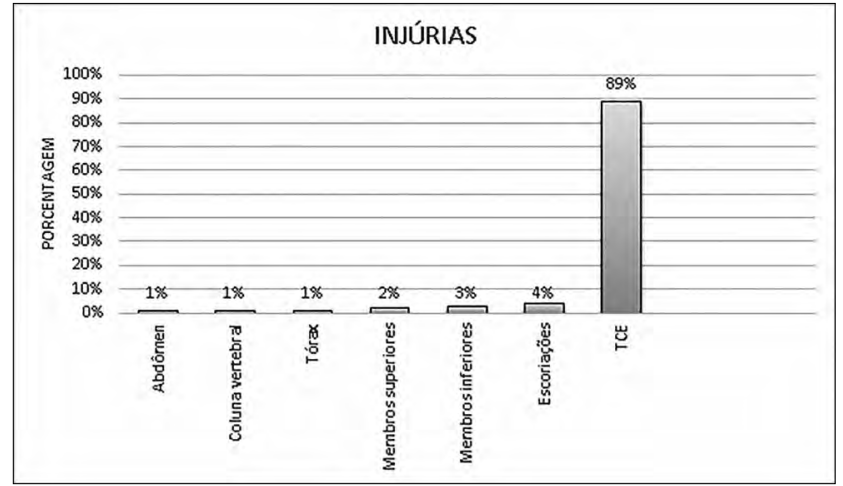

Fonte: autores.

Na Figura 6, observa-se as sequelas que ocorreram nos pacientes, em que $64 \%$ dos avaliados apresentaram Glasgow 15 (ou seja, sem déficit de consciência); $8 \%$ dos prontuários não forneciam informações pertinentes; $11 \%$ apresentaram Glasgow entre $12-14 ; 5 \%$ parestesia de nervos faciais; $5 \%$ paralisias; $3 \%$ Glasgow abaixo do escore número 12 ; $2 \%$ disfagia, disfasia e/ou afasia; e $2 \%$ amaurose e diminuição da acuidade visual.
Figura 6 - Distribuição do tipo de sequelas dos pacientes avaliados

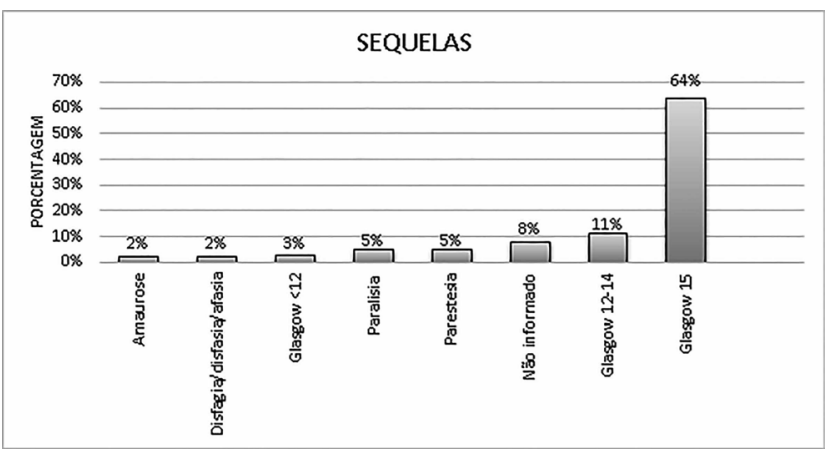

Fonte: autores.

\section{Discussão}

O grupo mais acometido de traumatismo em face foi o do sexo masculino, com $85 \%$. Isso se correlaciona com outros estudos ${ }^{6-9}$ e pode ser atribuído à maior exposição de indivíduos do sexo masculino a fatores de riscos (transitar em rodovias, praticar esportes de contato, ser maior consumidor de bebidas alcoólicas ou drogas) ${ }^{10}$.

A faixa etária deste estudo variou entre 20-29 anos (28\% dos casos), convergindo com alguns estudos presentes na literatura ${ }^{7,9,11,12}$. Isso se deve ao fato de esse grupo etário estar mais exposto a violência e conflitos socioeconômicos e por ter mais acesso a veículos automotores ${ }^{13,14}$.

As causas das fraturas faciais mudaram nos últimos anos, variando entre condições geográficas, socioeconômicas e culturais de cada região $0^{15,16}$. Numa revisão de artigos publicados entre 1980 e 2013 , os autore ${ }^{17}$ concluíram que a epidemiologia varia entre países e, até, no mesmo país. De modo geral, nos continentes americano, asiático e africano, a etiologia de traumas faciais que se sobressaiu foi o acidente de trânsito. No continente europeu, houve variação entre a supracitada e violência interpessoal. Já na Oceania, a violência foi a mais prevalente.

O acidente de trânsito ainda é o principal agente etiológico dos traumatismos faciais na maioria dos países ${ }^{7-9,12,18}$. Contudo, nos últimos anos, nota-se que a violência interpessoal vem aumentando gradativamente ${ }^{19-21}$; e outros autores mostram, em estudos no Brasil ${ }^{11,22}$, que a violência interpessoal já é o principal agente etiológico dos traumas em face. Isso pode ser explicado devido ao aumento da violência urbana no Brasil, que vem crescendo a cada ano. Este fator etiológico tende a aumentar, visto que, nos últimos anos, os investimentos em segurança pública e educação no país não foram significativos ${ }^{11}$.

Uma alternativa para reduzir o número de acidentes automobilísticos é a educação dos motoristas, com medidas para não se consumir bebidas alcoólicas ou drogas ilícitas quando dirigir, além de respeitar o limite de velocidade de cada rodovia. Também, 
é imperativo o cuidado das autoridades para com as rodovias e suas respectivas sinalizações ${ }^{16}$.

As quedas, segundo principal agente etiológico deste trabalho, são atribuídas, principalmente, aos extremos de idade. E este número tende a crescer, à medida que a população de idosos aumente ${ }^{23}$. Contudo, um estudo ${ }^{24}$ recente mostra que a população geriátrica vem sofrendo com a violência interpessoal nos últimos anos, tendo maior gravidade de trauma facial quando comparada a acidentes de trânsito e quedas.

A mandíbula foi o principal local acometido pelo trauma em face neste trabalho. Este achado converge com estudos realizados na Itália ${ }^{8}$, na Índia ${ }^{9}$, na Arábia Saudita ${ }^{15}$, na Nova Zelândia ${ }^{23}$ e também no Brasil $^{19}$.

A principal lesão concomitante ao trauma em face deste estudo foi o TCE. Este achado assemelha-se aos de outros autores ${ }^{10,25}$, em que a taxa de TCE variou de $5 \%$ a $20 \%$. Todavia, em uma pesquisa realizada em $2010^{6}, 25 \%$ dos pacientes estudados apresentaram alguma injúria associada à fratura dos ossos da face. A principal injúria associada difere do presente trabalho, em que aproximadamente $13 \%$ das vítimas apresentaram lesões em membros, seguidas de $11 \%$ de traumas no cérebro (que põe a vida do paciente em sérios riscos de óbito ou sequelas).

As injúrias no cérebro são relacionadas, geralmente, a trauma de alta velocidade (acidentes automobilísticos ou quedas) e associadas a fraturas severas de terço médio, fraturas combinadas ou zigomático-orbitais ${ }^{6,25}$.

Nesse contexto, foi realizado um estudo que mostrou que o acidente automobilístico permanece como principal fator etiológico quando se relacionam trauma em face e lesões corporais associadas. Além disso, 18\% dos casos de TCE resultaram em decorrência de acidentes automobilísticos. Por fim, quando ocorre TCE decorrente de fraturas faciais, o local mais acometido é o terço médio da face, em especial as seguintes fraturas: naso-órbito-etmoidal (NOE), Le Fort e de órbita ${ }^{26}$. Isso pode ser explicado devido à projeção anatômica da região, susceptível a traumas, além de estar próxima à região craniana, ocasionando danos sérios aos pacientes ${ }^{26,27}$.

Outro fator importante é que, se agrupar as fraturas orbitárias e dos ossos malares deste trabalho, este local será o mais acometido nos traumas faciais (35\% dos casos). Isso converge com outras pesqui$\operatorname{sas}^{26,27}$, em que essa associação esteve presente em aproximadamente $50 \%$ dos casos.

Neste estudo, $64 \%$ dos pacientes foram classificados com Glasgow 15, ou seja, sem deficiência neurológica. Em 8\% dos pacientes, não foram obtidas informações sobre ocorrência ou não de sequelas neurológicas.

Em 14\% dos pacientes deste estudo, houve dano de consciência. Este achado assemelha-se aos en- contrados na literatura, em que um quarto dos pacientes apresentou dano neurológico ${ }^{28}$.

Em relação à parestesia dos nervos cranianos, ela foi apresentada por 5\% dos pacientes. A literatura se mostra semelhante, pois $3 \%$ dos pacientes apresentaram parestesia temporária ${ }^{4}$.

Os traumas oriundos de acidentes automobilísticos geram forças que são suficientes para fraturar ossos da face, além de serem distribuídas por todo o corpo do paciente, ocasionando importantes lesões. Acidentes de alta cinemática geralmente causam lesões associadas em cabeça e pescoço (em média de 1 caso para cada 5 pessoas) $)^{29,30}$.

\section{Conclusões}

Os resultados obtidos com este estudo permitem concluir que, para a população estudada, mesmo com um subgrupo específico de traumatizados, o sexo masculino, a terceira década de vida e o acidente automobilístico ainda são mais prevalentes. O conjunto de ossos do terço médio, quando agrupados, foi a região anatômica mais acometida neste tipo de traumatismo que ocorre em crânio e face simultaneamente, e, ainda nesta condição de associação, o traumatismo cranioencefálico esteve presente na maioria dos casos, porém, somente $28 \%$ dos pacientes evoluíram com alguma sequela neurológica.

\section{Abstract}

Introduction: patients affected by craniofacial trauma may evolve into various sequelae. Objective: to identify the prevalence of neurological sequelae among patients with craniofacial trauma in a reference trauma service in southern Brazil. Subjects and method: a total of 1.385 medical records were analyzed, from which 169 (12\%) patients were selected with simultaneous skull and face trauma, considering the etiologic agent, origin, age and sex of the patient, and fracture location. Results: a rate of $85 \%$ of the individuals were men aged 31 through 40 years. The most prevalent etiological factors were car accidents (36\%), falls (22\%), and interpersonal violence $(21 \%)$. In this selected group, traumatic brain injury was present in $89 \%$ of the cases. In $64 \%$ of the population, there were no neurological sequelae, 28\% presented some type of sequelae, and $8 \%$ of the medical records did not contain complete information. Conclusion: for this population studied, even with a specific subgroup of traumatized subjects, the male sex, the third decade of life, and car accidents are the most prevalent factors. In addition, the most affected anatomic region is the set of bones in the middle third of the face and, in this condition of association, traumatic brain injury was present in the great majority of cases, but only $28 \%$ of them evolved into some type of neurological sequelae.

Keywords: Epidemiology. Maxillofacial trauma. Traumatology. 


\section{Referências}

1. Wulkan M, Parreira Jr JG, Botter DA. Epidemiology of facial trauma. Assoc Med Bras 2005; 51(5):290-5.

2. Nascimento EN, Gimeniz-Paschoal SR. Os acidentes humanos e suas implicações fonoaudiológicas: opiniões de docentes e discentes sobre a formação superior. Ciênc Saúde Colet 2008;13(2):2289-98.

3. Oliveira E, Lavrador JP, Santos MM, Lobo Antunes J. Traumatismo crânio-encefálico: abordagem integrada. Acta Med Port 2012; 25(3):179-92.

4. Salentijn EG, Van Der Bergh B, Forouzanfar T. A ten-year analysis of midfacial fractures. J Craniomaxillofac Surg 2013; 41(7):630-6.

5. Jerônimo SA, Creôncio SCE, Cavalcanti D, Moura JC, Ramos RA, Paz AM. Fatores relacionados ao prognóstico de vítimas de traumatismo carnioencefálico: uma revisão de bibliográfia. Arq Bras Neurocir 2014; 33(3):165-9.

6. Thorén H, Snäll J, Salo J, Suominen-Taipale L, Kormi $\mathrm{E}$, Lindqvist $\mathrm{C}$, et al. Occurrence and types of associated injuries in patients with fractures of the facial bones. J Oral Maxillofac Surg 2010; 68(4):805-10.

7. Cavalcanti AL, Bezerra PKM, Oliveira DM, Granville-Garcia AF. Maxillofacial injuries and dental trauma in patients aged 19-80 years, Recife, Brazil. Rev Española de Cirugía Oral y Maxilofacial 2010; 32(1):11-6.

8. Bonavolontà P, Dell'aversana Orabona G, Abbate V, Vaira LA, Lo Faro C, Petrocelli M, et al. The epidemiological analysis of maxillofacial fractures in Italy: The experience of a single tertiary center with 1720 patients. J Craniomaxillofac Surg 2017; 45(8):1319-26.

9. Monodh P, Prabhu Shankar D, Pradeep D, Santhosh R, Murugan A. Incidence and patterns of maxillofacial traumaa retrospective analysis of 3611 patients-an update. Oral Maxillofac Surg 2016; 20(4):377-83.

10. Silva JJL, Lima AAAS, Dantas TB, Da Fronta MHA, Parente RV, Lucena ALSPN. Fratura de mandíbula: estudo epidemiológico de 70 casos. Rev Bras Cir Plast 2011; 26(4):645-8.

11. Macedo JL, Camargo LM, Almeida PF, Rosa SC. Perfil epidemiológico do trauma de face dos pacientes atendidos no pronto socorro de um hospital público. Rev Col Bras Cir 2008; 35(1):9-13.

12. Ribeiro AL, Gillet LCS, Vasconcelos HG, Rodrigues LC, Pinheiro JJV, Alves-Júnior SM. Facial fractures: large epidemiologic survey in northern Brazil reveals some unique characteristics. J Oral Maxillofac Surg 2016; 74(12):2480. e1-2480.e12.

13. Silva JJL, Lima AAAS, Torres SM. Fraturas de face: análise de 105 casos. Rev Bras Cir Craniomaxilofac 2009; 12(1):1620

14. Moura MTFL, Daltro RM, Almeida TF. Traumas faciais: uma revisão sistemática da literatura. RFO UPF 2016; 21(3):331-

15. Abdullah WA, Al-Mutairi K, Al-Ali Y, Al-Soghier A, AlShnwani. Patterns and etiology of maxillofacial fractures in Riyadh City, Saudi Arabia. Saudi Dent J 2013; 25(1):33-8.

16. Adhikari RB, Karmacharya A, Malla N. Pattern of mandibular fractures in western region of Nepal. Nepal J Med Sciences $2012 ; 1(1): 45-8$.

17. Boffano P, Kommers SC, Karagozoglu KH, Forouzanfar T. Aetiology of maxillofacial fractures: a review of published studies during the last 30 years. Br J Oral Maxillofac Surg 2014; 52(10):901-6
18. Gonzales E, Pedemonte C, Vargas I, Lazo D, Pérez H, Canales M, et al. Fracturas faciales en un centro de referencia de traumatismos nivel I. Estudio descriptivo. Rev Española de Cirugía Oral y Maxilofacial 2015; 37(2):65-70.

19. Noronha Filho OL, Valente C, Kozlowsky K. Etiologia e incidência das fraturas faciais: análise de 152 casos. Rev Univ Vale do Rio Verde 2012; 10(1):117-23.

20. Bortoli MM, De Conto F, Eidt JMS, Engelmann JL, Rocha FD. Trauma maxilofacial: avaliação de 1385 casos de fraturas de face na cidade de Passo Fundo - RS. Rev Cir Traumatol Buco-Maxilo-Fac 2014; 14(2):87-94.

21. Possebon APR, Faot GGF, Pinto LR, Leite FRM, Torriani MA. Etiology, diagnosis, and demographic analysis of maxillofacial trauma in elderly persons: A 10-year investigation. $\mathrm{J}$ Cranio-Maxillofac Surgery 2017; 45(12):1921-6.

22. Zamboni RA, Wagner JCB, Volkweis MR, Gerhardt EL, Buchmann EM, Bavaresco CS. Levantamento epidemiológico das fraturas de face do Serviço de Cirurgia e Traumatologia Bucomaxilofacial da Santa Casa de Misericórdia de Porto Alegre - RS. Rev Col Bras Cir 2017; 44(5):491-7.

23. Moore BK, Smit RB, Colquhoun AN, Thomson WM. Maxillofacial fractures at Waikato Hospital, New Zealand: 2004 to 2013. N Z Med J 2015; 128(1426):96-102.

24. Cillo Jr JE, Holmes TM. Interpersonal violence is associated with increased severity of geriatric facial trauma. J Oral Maxillofac Surgery 2016; 74(5):1023. e1-1023.e7.

25. Tino MT, Andrade FA, Gonçalves AJ, Freitas RR. Epidemiologia do trauma maxilofacial num hospital universitário terciário da cidade de São Paulo. Rev Bras Cir Cabeça Pescoço 2010; 39(2):139-45.

26. Eidt JMS, De Conto F, Bortoli MM, Engelmann JL, Rocha FD. Associated injuries in patients with maxillofacial trauma at the Hospital São Vicente de Paulo, Passo Fundo, Brazil. J Oral Maxillofac Res 2013; 4(3):e1.

27. De Conto F, Rhoden R, Santos RS, Nicolini IC. Levantamento epidemiológico das fraturas de face no hospital São Vicente de Paulo, Passo Fundo, RS. RFO UPF 2003; 8(2):80-4.

28. Salentijn EG, Collin JD, Boffano P, Forouzanfar T. A ten year analysis of the traumatic maxillofacial and brain injury patient in Amsterdam: Complications and treatment. J Craniomaxillofac Surg 2014; 42(8):1717-22.

29. Follmar KE, Debruijn M, Baccarani A, Bruno AD, Mukundan S, Erdmann D, et al. Concomitant injuries in patients with panfacial fractures. J Trauma 2007; 63(4):831-5.

30. Thorén H, Schaller B, Suominen AL, Lindqvist C. Occurrence and severity of concomitant injuries in other areas than the face in children with mandibular and midfacial fractures. J Oral Maxillofac Surg 2012; 70(1):92-6.

\section{Endereço para correspondência:}

Cassian Taparello

Rua Francisco Costa, 109, Centro

99670-000, Ronda Alta, Rio Grande do Sul, Brasil

Telefone: (54) 996029113

E-mail: cassian.taparello@gmail.com

Recebido: 21/06/18. Aceito: 04/07/18. 